\title{
Conceptual activation of distractors during selection is not sufficient to produce negative priming
}

\author{
VERONICA J. DARK and PATRICIA A. SCHMIDT \\ Iowa State University, Ames, Iowa
}

\begin{abstract}
Negative priming (NP) occurs when responses are slower because the targets were distractors on the preceding trial. Word-naming NP occurs only with words that have been presented repeatedly as targets; novel words do not show NP. The activation-inhibition explanation is that representations of repeated-word distractors are activated already and must be inhibited; the inhibition carries over to the next trial. If this explanation is correct, novel-word NP should occur if the word is semantically primed (thus activating its representation) before it occurs as a distractor. In two experiments, there was NP for words from a repeated set, and the magnitude of NP increased when the same word could occur as a target on consecutive trials. There was positive, rather than negative, priming for novel-word targets that had been semantically primed as distractors. Either the activation from semantic priming was not sufficiently strong to require inhibition, or the activation-inhibition hypothesis does not refer to activation of conceptual representations.
\end{abstract}

Responses to a just ignored stimulus often are slower than responses either to a just responded to stimulus or to a novel stimulus. Tipper (1985) coined the phrase negative priming (NP) to refer to this apparent bias against the reprocessing of previously irrelevant information. NP is found with a wide variety of experimental procedures and there are a number of competing explanations of it (for reviews, see Fox, 1995; May, Kane, \& Hasher, 1995; Neill, Valdes, \& Terry, 1995). The present research is concerned primarily with the activation-inhibition explanation of NP in a naming task.

In a typical naming task, participants engage in a selective-attention task in which multiple words are presented on each trial. The target is defined by some physical characteristic, such as color, and the task is to name the target. Sometimes a pair of trials constitutes an ignored repetition (IR) couplet. In an IR couplet, a given stimulus is presented on consecutive trials, first as a to-be-ignored distractor, and then as a to-be-named target. That is, the target on trial $N$ (referred to as the probe trial) was a distractor on trial $N-1$ (referred to as the prime trial). NP occurs when the response on IR probe trials is slowed relative to the response on some baseline trial (e.g., the prime trial) in which the distractor on the preceding trial was a stimulus other than the current target.

The theoretical explanation of NP that is the focus of the present research is that NP reflects inhibition, or re-

\footnotetext{
We thank Elizabeth Andre, Karla Benson, Sharon Chambers, Brian Crabb, Amanda Easton, and Luke Rossielle for help with data collection. We thank Elaine Fox and David Strayer and an anonymous reviewer for comments on an earlier version of the manuscript. Portions of the data were presented at the November 1996 meeting of the Psychonomic Society. Correspondence concerning this article may be addressed to V. J. Dark, Department of Psychology, Iowa State University, Ames, IA 50011-3180 (e-mail: vjdark@iastate.edu).
}

sponse blocking (e.g., Tipper \& Cranston, 1985). The activation-inhibition explanation assumes that both the target and the distractor(s) presented on the IR prime trial are processed and that their representations become activated. Because the distractor's representation is activated but is to be ignored, it must be inhibited on the prime trial; the inhibition is accomplished by blocking access of the distractor's representation to the response mechanism so that the correct target response can be made. NP occurs when this inhibition carries over to a subsequent trial. That is, when the distractor from the IR prime trial becomes the target on the IR probe trial, responding is slowed because the inhibition associated with the distractor on the prime trial must be overcome in order for the appropriate response to be made to the target on the probe trial (Tipper \& Cranston, 1985)

Malley and Strayer (1995) further developed the activation-inhibition explanation by showing that inhibition, which is necessary for NP, occurs only under conditions in which the representations of distractors are highly activated already. They noted that in the typical NP study, stimuli are selected from a limited set of items with a varied mapping procedure such that specific stimuli are sometimes targets and sometimes distractors. Thus, the representation of the distractor on each trial is highly activated already because it has been presented repeatedly as a target. Because it is highly activated already, the representation of the distractor must be inhibited so that the response to the target can be made.

Malley and Strayer (1995) measured NP as the difference in latency between the response on the prime trial and the response on the probe trial in an IR couplet. The critical manipulation used to test the stimulus-repetition version of the activation-inhibition hypothesis was whether the words in the IR couplet were from a set of repeated words, or whether they were experimentally novel, occur- 
ring for the first time in the IR couplet. The importance of stimulus repetition to NP was demonstrated by the fact that whereas repeated-word IR couplets showed NP (slower responses to the probe trials), novel-word IR couplets did not. In fact, sometimes novel-word IR couplets showed reliable positive priming (Malley \& Strayer, 1995, Experiment 5; Strayer \& Grison, 1999, Experiment 2). Malley and Strayer argued that stimulus repetition produces high activation, and that the inhibition responsible for the NP effect is needed only when distractor representations are already highly activated.

Prime-trial distractors that produced NP in Malley and Strayer (1995) had highly activated representations as the result of repeated prior processing. The present research was designed to test the generality of the activationinhibition hypothesis by examining a situation in which a single episode of semantic priming, rather than multiple episodes of identity priming, was used to make the conceptual representation of a novel word active prior to the word's being presented in an IR couplet. We assumed that the representation of a novel word could be activated via automatic spreading activation from an associatively related word. We reasoned that if the target on the trial preceding a novel-word IR couplet was associatively related to the prime-trial distractor, the distractor's conceptual representation would be activated already when the prime-trial display occurred. As a result of its already active status, the representation of the distractor would need to be inhibited and this inhibition would carry over to the probe trial. Thus, we reasoned that if the activationinhibition hypothesis refers to the activation of the conceptual representation of a stimulus, a semantic priming procedure could be used to produce NP with novel words.

\section{EXPERIMENT 1}

On each trial, participants saw both a target, which was presented in red, and a distractor, which was presented in white. On most trials, both words were drawn from a set of eight repeated words, but on some trials either the distractor or the target was drawn from a novelword set. NP for repeated words was examined in standard IR couplets. NP was computed by subtracting the response latency to the repeated-word IR prime trials from the response latency to the repeated-word IR probe trials.

NP for novel words was computed from performance in triplets (sequences of three trials). Each triplet consisted of a context trial with a novel-word target followed by a novel-word IR couplet (a prime trial with a novelword distractor followed by a probe trial with the novel word as the target). There were two types of triplets. In a related triplet, the target on the context trial was associatively related to the distractor on the prime trial of the subsequent IR couplet. An example of a related triplet is the following three pairs: DOCTOR-flood, CLOCK-nurse, NURSE-valley. In this example, targets are printed in uppercase letters whereas distractors are printed in lower- case letters, and repeated words are printed in italics whereas novel words are not. Notice that the second and third trials consist of an IR couplet, with the novel word "nurse" occurring first as a distractor and then as a target. The first trial in the triplet functions as a related context trial because the target, the novel word "doctor," is associatively related to the distractor "nurse" on the subsequent IR prime trial. In an unrelated triplet, the target on the context trial was not associatively related to the distractor on the subsequent IR prime trial. In the preceding example, substituting LION-flood for DOCTOR-flood illustrates an unrelated triplet.

Novel-word NP was computed by subtracting the response latency to related IR probes from the response latency to unrelated IR probes. The logic for this computation was as follows: As a result of the response to the target on the context trial, the representation of the distractor on related IR prime trials would be more active than the representation of the distractor on unrelated IR prime trials. The enhanced activation of the distractor's representation on the related prime trial would interfere more with target processing such that target processing would need to be inhibited. The inhibition would carry over to the probe trials, leading to a slower response on the related IR probe trials than on the unrelated IR probe trials.

On the basis of the activation-inhibition hypothesis, we expected to find reliable NP for repeated words and for related novel words. Although May et al. (1995) pointed out that there is no systematic relationship between the level of interference on IR prime trials and the level of NP on IR probe trials (see also Fox, 1995), the results of prior research in our lab (e.g., Dark, Vochatzer, \& VanVoorhis, 1996) led us to expect prime-trial interference in which the responses on the related IR prime trials would be slower than the responses on the unrelated IR prime trials.

\section{Method}

Participants. Twenty undergraduate students ( 7 males and 13 females) enrolled in introductory psychology courses served as the participants in exchange for extra credit. All participants reported normal or corrected-to-normal vision and fluency in English.

Stimuli. A base list of stimurti was constructed in which there were 28 blocks of 24 trials each ( 672 total trials). Sixteen trials within each block were repeated-word trials in which both the target and the distractor were selected from a set of 8 common nouns, four to six letters long. Eight of these trials were fillers (i.e., trials unrelated to any other trial), and the other 8 trials comprised four IR couplets in which the distractor on the prime trial was the target on the probe trial. Except for the stimuli in IR couplets, the target and the distractor on each trial were selected randomly from the set with the constraint that no word could appear on two consecutive trials.

Eight trials within a block were novel-word trials in which either the target or the distractor was a novel word ranging from three to nine letters long. Two trials were novel-word fillers in which the target was a novel word and the remaining six trials comprised two triplets, one related and one unrelated. Each triplet consisted of a context trial followed by a novel-word IR couplet. On related triplets, the target on the context trial was a novel word associatively related to the novel-word distractor in the prime trial of the subsequent IR couplet. On unrelated triplets, the target on the context trial was 
a novel word not associatively related to the subsequent distractor. In triplets, the distractor on the context trial, the target on the prime trial, and the distractor on the probe trial all were selected from the repeated-word set.

The order of trial types varied randomly within a block with the constraint that a repeated-word filler trial preceded each repeatedword IR couplet and each novel-word triplet. All participants saw the same ordering of trials. A second list was constructed from the base list so that related triplets on one list were unrelated triplets on the other. Half of the participants were presented each list. The same practice block of 20 repeated-word trials (containing four IR couplets) preceded the experimental trials in each list

Procedure. Each trial began with the presentation of two white plus signs in the center of the screen. After $500 \mathrm{msec}$, the word pair was presented: one word was red and one was white, one word was presented above and one below the plus signs. The location of the red word was randomly determined on each trial for each person. The word pair was presented for $150 \mathrm{msec}$ and was then removed leaving only the plus signs, which remained until a response was made. The screen then went blank for $500 \mathrm{msec}$ and the next trial began. Trials occurred in a continuous stream with nothing indicating when a trial was part of a couplet or triplet. That is, from the participant's view, each trial was unrelated to any other trial. A participant-terminated rest period occurred after every 140 trials (seven blocks).

Participants were instructed to verbally identify the red word as quickly and as accurately as possible. Response latency was recorded with a voice key and microphone. Accuracy was recorded by the research assistant. Displays were viewed from approximately $40 \mathrm{~cm}$. The entire display subtended approximately $3^{\circ}$ of visual angle vertically, and no more than $4^{\circ}$ horizontally. Stimulus presentation and response recording were controlled by a Zenith 386 computer programmed using Micro Experimental Laboratory software (Schneider, 1988).

\section{Results}

An alpha level of .05 was used in all inferential analyses in both experiments, and only correct responses were included in the analyses. ${ }^{1}$

Voice key errors occurred on $2.3 \%$ of the trials. Each participant's mean response time and standard deviation were computed separately for novel words and for repeated words. Outliers were defined as any response greater than three standard deviations from a participant's mean. Outliers were replaced with the cutoff value; less than $1 \%$ of the data were replaced.

Repeated-word NP was measured as the latency difference between responses on the probe and the prime trials in the IR couplets (i.e., repeated-word prime latency minus the repeated-word probe latency). Both the latencies and the difference scores are shown in Table 1. The latency to the repeated-word fillers also is shown. Just as in the results of Malley and Strayer (1995), responses to repeated-word fillers and to repeated-word prime trials were nearly identical, indicating that the prime trials were an appropriate baseline against which to compare the probe trials. A one-tailed test against zero showed that there was reliable NP on the repeated-word trials $[t(19)=-2.11, S E=2.23]$.

The latencies and difference scores from the novelword trials also are shown in Table 1. Prime-trial interference was measured as the latency difference between re-
Table 1

Mean Response Latencies and Difference Scores (in Milliseconds) for Each Type of Trial in Experiments 1 and 2

\begin{tabular}{|c|c|c|c|c|}
\hline & \multicolumn{2}{|c|}{ Experiment $1(n=20)$} & \multicolumn{2}{|c|}{ Experiment $2(n=22$} \\
\hline & $M$ & $S E$ & $M$ & $S E$ \\
\hline \multicolumn{5}{|c|}{ Repeated-Word Trials } \\
\hline Filler & 528.5 & 9.0 & 541.3 & 6.7 \\
\hline Prime & 527.5 & 9.2 & 540.2 & 6.9 \\
\hline Probe & 532.2 & 9.3 & 549.6 & 6.3 \\
\hline Difference & $-4.7^{*}$ & 2.2 & $-9.4^{*}$ & 2.0 \\
\hline \multicolumn{5}{|c|}{ Novel-Word Prime Trials } \\
\hline Related & 543.6 & 9.3 & 562.6 & 7.3 \\
\hline Unrelated & 549.7 & 9.9 & 561.8 & 8.1 \\
\hline Difference & -6.1 & 4.1 & +0.8 & 1.8 \\
\hline \multicolumn{5}{|c|}{ Novel-Word Probe Trials } \\
\hline Related & 555.2 & 11.1 & 581.5 & 9.9 \\
\hline Unrelated & 541.6 & 10.7 & 569.1 & 8.4 \\
\hline Difference & $+13.6^{*}$ & 6.1 & $+12.4^{*}$ & 5.5 \\
\hline
\end{tabular}

*Reliably different from zero, $p<.05$.

sponses on the unrelated novel-word IR prime trials and the related novel-word IR prime trials. Although the difference was in the predicted direction, there was no reliable evidence of inhibition in the responses to the related IR prime trials $[t(19)=-1.49, S E=4.09]$.

Novel-word NP was measured as the latency difference between responses on the unrelated novel-word IR probe trials and the related novel-word IR probe trials. There was no evidence of novel-word NP on the related IR probe trials, but there was reliable positive priming $[t(19)=+2.24, S E=6.07]$.

\section{Discussion}

Two predictions derived from our interpretation of the activation-inhibition hypothesis were tested in Experiment 1 . The first prediction, that there would be repeatedword NP, was supported. The magnitude of the NP was smaller in absolute magnitude $(-4.7 \mathrm{msec})$ than the typical range of 10-25 msec reported by May et al. (1995), but the effect was reliable. The second prediction, that there would be novel-word NP, was not supported. There was facilitation $(+13.6 \mathrm{msec})$, rather than inhibition, of the responses on related IR probe trials as opposed to unrelated trials. Additionally, we looked for evidence of interference in the responses to related IR prime trials. There was no evidence of reliable interference. May et al. suggested that the lack of a relationship between prime-trial interference and probe-trial NP is evidence that inhibition of the distractor occurs after target selection of the prime trial. The lack of interference in the present research, together with the finding of positive priming, suggests that although the conceptual representation of the distractor on the novel-word IR prime trial was activated, it was not inhibited.

The positive priming found on the novel-word IR probe trials was unexpected (NP was predicted) and is quite different from that sometimes found by Strayer and colleagues (Malley \& Strayer, 1995, Experiment 5; Strayer \& Grison, 1999, Experiment 2). Their positive priming 
was a form of repetition priming in which the response to a novel-word target was enhanced if the word had first occurred as a distractor on the preceding trial. That is, their novel-word priming constituted the difference in response latency between responses on novel-word IR probe trials and responses in a novel-word control condition in which the current target was not the same as the preceding distractor. In the present research, the positive novelword priming constituted the difference in response latencies to two IR probe targets, one in a related triplet and one in an unrelated triplet. In each case, the target was the distractor on the preceding trial. The only difference between the related and unrelated novel-word IR probe trials was whether or not the context target was related to the prime-trial distractor. We assumed that the difference in context produced a difference in the conceptual representation of the distractor on the IR prime trial such that the representation was activated on related trials.

The fact that positive, rather than negative, priming was found for related novel-word IR probe trials can be interpreted in a number of ways (as will be addressed in the General Discussion). Before attempting to relate the quite unexpected findings to the activation-inhibition hypothesis, however, we thought it necessary to replicate them.

\section{EXPERIMENT 2}

Experiment 2 was a replication of Experiment 1 with the addition of attended repetition $(A R)$ couplets in which the same repeated-word target was presented on consecutive trials (e.g., FLOOD-dream, clock-FLOOD). The AR couplets were added in an attempt to make the procedure as conducive to finding reliable NP effects as possible. Although our reading of the activation-inhibition hypothesis indicated no apparent reason why the inclusion of consecutively repeated targets should influence the magnitude of the NP effect, we noted that Malley and Strayer (1995) and Strayer and Grison (1999) included a high proportion of AR trials. In addition, May et al. (1995) and Kane, May, Hasher, Rahhal, and Stoltzfus (1997) pointed out that the inclusion of such trials was associated with larger NP effects. We reasoned that if the inclusion of AR trials makes it easier to detect NP, perhaps we would find NP for related novel words.

\footnotetext{
Method

Participants. Twenty-two undergraduate students ( 4 males and 18 females) enrolled in introductory psychology courses served as the participants in exchange for extra credit. All participants reported normal or corrected-to-normal vision and fluency in English. None had participated in the prior experiment.

Stimuli and Procedure. The stimuli and procedure in this experiment were identical to those in Experiment 1 with one exception: 56 repeated-word AR couplets were added, 2 couplets per block. Each AR couplet was preceded by an additional repeatedword filler. Thus, there were 32 trials per block ( 896 total trials).
}

\section{Results}

Voice key errors occurred on $2.4 \%$ of the trials. Outliers were treated as in Experiment 1; less than $1 \%$ of the data were replaced with the cutoffs.

Table 1 shows the mean response latencies and the IR prime-IR probe difference score for repeated-word trials. A one-tailed test against zero showed that there was reliable repeated-word NP $[t(21)=-4.62, S E=2.06]$, and the magnitude $(-9.4 \mathrm{msec})$ was twice that found in Experiment 1.

The latencies and difference scores for the novel-word trials also are shown in Table 1 . There was no evidence of prime-trial interference; that is, responses to the related novel-word IR prime trials were not reliably different from responses to the unrelated trials $[t(21)=$ $+0.44, S E=1.92]$. There was no evidence of novelword NP, but there was novel-word positive priming; that is, there was reliable facilitation $(+12.4 \mathrm{msec})$ on the related novel-word IR probe trials relative to the unrelated trials $[t(21)=+2.24, S E=5.34]$.

An attended-repetition difference score was computed as the difference in latency between responses to the first trials in AR couplets (540.4 msec, $S E=6.19$ ) and responses to the second trials $(554.4 \mathrm{msec}, S E=7.00)$. Somewhat unexpectedly, the difference was negative. That is, the second response was $14 \mathrm{msec}$ slower than the first $[t(21)=-4.10, S E=3.41]$.

\section{Discussion}

The inclusion of AR couplets did not change performance on the novel-word trials, which showed the same pattern found in Experiment 1: There was positive priming, rather than NP, for related IR probes, and there was no evidence of prime-trial interference. In the General Discussion we will consider what this replicable outcome may indicate about the activation-inhibition hypothesis.

The inclusion of AR couplets did change performance on the repeated-word trials: The magnitude of the NP effect was larger in Experiment 2 than in Experiment 1 and was at the lower end of the typical range reported by May et al. (1995). The increase suggests that the magnitude of NP may be influenced by the inclusion of trials on which the same stimulus is repeated immediately as a target. Why this might be the case is not clear if one explains NP solely in terms of the activation-inhibition hypothesis: We do not see how the inclusion of consecutively repeated targets could affect the level of activation of the conceptual representations of the words in the repeated-word set. Therefore, the fact that inclusion of AR couplets enhances the level of NP suggests that something more than just distractor activation (and inhibition) is contributing to the NP effect with repeated words.

May et al. (1995) suggested that the inclusion of consecutively repeated targets is associated with the use of an episodic-retrieval strategy (Neill et al., 1995; Neill, Valdes, Terry, \& Gorfein, 1992), that also contributes to 
NP. According to May et al., when targets are repeated at a high rate, the participant builds an expectancy that the target will be repeated and, as a result of this expectancy, employs a strategy of using retrieval of the prior episode to facilitate the response (see also Kane et al., 1997). However, because there were so few consecutively repeated targets in Experiment 2, it seems unlikely that their occurrence would have caused participants to adopt an episodic-retrieval strategy. The fact that responses to repeated targets were actually slower than responses to nonrepeated targets also argues against this explanation.

The finding of inhibition on AR trials when a target is repeated does not replicate results reported in the literature either that there is no difference in response time to consecutively repeated targets from a repeated-word set (Malley \& Strayer, 1995, Experiments 3 and 4) or that facilitation occurs for such targets (Malley \& Strayer, 1995, Experiment 5; Stadler \& Hogan, 1996). An important difference between our procedure and the procedures in those studies, however, is that consecutively repeated target sequences were very common in those experiments. In Malley and Strayer (Experiment 5), for example, consecutively repeated targets occurred on fully half of the trials. Consecutively repeated targets occurred on fewer than $7 \%$ of the trials in the present research. Although the participants were informed of the possibility of consecutively repeated targets in the instructions and were given an example in the practice, such trials were so infrequent that the participants may have hesitated when they were required to say the same word twice in a row. ${ }^{2}$

\section{GENERAL DISCUSSION}

In two experiments we failed to find NP with novelword IR couplets even though steps were taken to activate the representation of the distractor on the prime trial. We reasoned that spreading activation from an associatively related word would activate the representation of a novel-word distractor so that the representation would be functionally similar to that of a repeated-word distractor and that, if the activation-inhibition hypothesis were true, the representation would be inhibited. That is, we predicted that by incorporating a semantic priming manipulation into a situation that produced NP for repeated words, we could produce novel-word NP. There was no support for the prediction. Rather, there was positive priming of the responses to related (as opposed to unrelated) novel-word IR probe targets. The results lead to the following conclusion concerning the activationinhibition hypothesis: Either the conceptual activation produced by an associatively related context is not sufficiently strong to require inhibition on the IR prime trial, or the activation referred to in the activation-inhibition hypothesis is not the activation of the conceptual representation of a stimulus that results from spreading activation. This conclusion, of course, rests on two assumptions: first, that the semantic priming procedure worked to activate the conceptual representations of the related IR prime distractors; and second, that those representations were used in the naming task. If either of those assumptions was not met, the results could not be interpreted in terms of the activation-inhibition hypothesis.

Consider the possibility, suggested by a reviewer, that spreading activation did not occur, so that there actually was no difference in the activation of the conceptual representations of the distractors on the IR prime trials as a function of relatedness. In this view, the positive priming effect might reflect the impact of some other semantic priming mechanism, such as expectancy or a postlexical matching process (see Neely, 1991), operating directly between the context-trial target and the IR probe-trial target, and over the intervening IR prime trial. If this were the case, then the results would say nothing about the activationinhibition hypothesis.

Because our experimental procedure was based on the assumption that spreading activation would occur, it was not designed to assess the possible contribution of other mechanisms to performance. Therefore, we cannot rule out the possibility that the observed positive priming reflects the operation of other mechanisms. However, we can point out that the empirical evidence for reliable positive priming is mixed when there is an intervening event (in this case the IR prime trial) between a semantic prime and its target (Neely, 1991), and that when such effects are found, they have been used to argue for (rather than against) a spreading activation account of the effect (e.g., Joordens \& Besner, 1992; McNamara, 1992a,1992b; also see Masson, 1995). In addition, although Neely (1991) presented convincing arguments that the mechanism of spreading activation could not by itself explain all of the phenomena identified in the semantic priming literature, his theoretical account of the phenomena was that the other mechanisms operated in addition to spreading activation. We note that as long as spreading activation is assumed to occur, even if other mechanisms also operate, there should be a difference in the activation of the conceptual representations of related and unrelated distractors on the IR prime trials, and the situation is relevant to the activation-inhibition hypothesis.

Consider next the possibility, also suggested by a reviewer, that the present results are not relevant to the activation-inhibition hypothesis because the naming task does not require access to the conceptual representations that are activated by the related context. Tipper, Weaver, and Houghton (1994) suggested that only response-relevant characteristics of a distractor are inhibited. If the naming task can be carried out without accessing conceptual representations, there would not be any need to inhibit those representations, even if they were highly activated, and there would be no novel-word NP in our task. Although we agree that the present results show activation without inhibition for novel words, there are two reasons why we do not think that the problem lies in the use of the naming task. First, the occurrence of positive seman- 
tic priming indicates that the naming response is sensitive to activation of conceptual representations. Second, there is NP in the naming task with the repeated words.

Given that the novel-word results are relevant to the activation-inhibition hypothesis, one may ask how the hypothesis might be modified to handle the results. One interpretation of the results is that they demonstrate the importance of level of activation. The activation-inhibition hypothesis implicitly assumes that there is some minimal level of activation needed before inhibition is necessary. Strayer and Grison (1999) found no NP on IR probe trials for words that had been repeated many times, but only as distractors. Only repeated presentations of a word as a target led to reliable NP on IR probe trials. Strayer and Grison interpreted their findings in terms of level of activation: They assumed that the processing of targets produced higher levels of activation than did the processing of distractors. If this interpretation is accurate, a similar situation might be occurring in the present results. It is possible that the level of activation produced by an associative prime is not sufficient to warrant inhibition and this may explain why positive rather than NP was obtained on related novel-word probe trials. In this view, our results simply show that the activation-inhibition hypothesis needs to be modified to acknowledge explicitly that some to-be-specified level of activation greater than that produced by a semantic prime must be reached before inhibition is required.

An alternative interpretation of the results is that they show that the representation referred to by the activationinhibition hypothesis is not the conceptual representation activated by an associative context. The question then becomes, "Just what is the nature of the representation that is inhibited?" May et al. (1995) described the arguments by which empirical results have been used to rule out the contribution of inhibited perceptual representations and inhibited response-related representations to NP and to suggest that it is conceptual representations that are inhibited. If the present results are used to rule out the contribution of inhibition of conceptual representations, no standard type of representation remains to be inhibited. The logic upon which these arguments rest breaks down, however, if more than one level of representation is involved in NP.

In the typical NP study, there is a small set of repeated stimuli. As a result, multiple types of representations of each stimulus should be highly active (i.e., there is activation of the perceptual, conceptual, and responserelated representations) and the participant faces a tough discrimination problem in deciding which representation(s) should guide each particular response. It may be that the constellation of activated representations is important, and that different combinations of representations could contribute to NP under different circumstances with no one type of representation sufficient to always produce it. Such a constellation of active representations might occur only with repeated processing of a stimulus as a target.

We note a similarity between the situation just described and a discussion that occurred as part of the controversy over early versus late selection in attention (see Johnston $\&$ Dark, 1986). At issue was the nature of the processing received by "unattended" stimuli. As noted by Kahneman and Treisman (1984), results from studies using filtering paradigms, in which a wide range of stimuli were presented, and results from studies using stimulus-set paradigms, in which a small set of stimuli were used repeatedly, presented quite different pictures about the nature of "unattended" processing. Repeatedly presented stimuli appeared to be more fully processed when "unattended" than did novel stimuli. In the same vein, NP as a phenomenon might be limited to repeated stimuli. Explanations developed from the typical NP experiment simply may not generalize to the processing of novel stimuli, even those with active conceptual representations. Our failure to find NP with related novel words suggests an important role for target repetition, and not just activation, in requiring inhibition.

\section{REFERENCES}

Dark, V. J., Vochatzer, K. G., \& VanVoorhis, B. A. (1996). Semantic and spatial components of selective attention. Journal of Experimental Psychology: Human Perception \& Performance, 22, 63-81.

Fox, E. (1995). Negative priming from ignored distractors in visual selection: A review. Psychonomic Bulletin \& Review, 2, 145-173.

JOHNSTON, W. A., \& DARK, V. J. (1986). Selective attention. Annual Review of Psychology, 37, 43-75.

JoORDENS, S., \& BESNER, D. (1992). Priming effects that span an intervening unrelated word: Implications for models of memory representation and retrieval. Journal of Experimental Psychology: Learning, Memory, \& Cognition, 18, 483-491.

Kahneman, D., \& Treisman, A. (1984). Changing views of attention and automaticity. In R. Parasuraman \& D. R. Davies (Eds.), Varieties of attention (pp. 29-61). Orlando, FL: Academic Press.

Kane, M. J., May, C. P., Hasher, L., Rahhal, T., \& Stoltzfus, E. R. (1997). Dual mechanisms of negative priming. Journal of Experimental Psychology: Human Perception \& Performance, 23, 632-650.

MALLEY, G. B., \& STRAYER, D. L. (1995). Effect of stimulus repetition on positive and negative identity priming. Perception \& Psychophysics, 57, 657-667.

Masson, M. E. J. (1995). A distributed memory model of semantic priming. Journal of Experimental Psychology: Learning. Memory, \& Cognition, 21, 3-23.

MaY, C. P., Kane, M. J., \& Hasher, L. (1995). Determinants of negative priming. Psychological Bulletin, 118, 35-54.

MCNamara, T. (1992a). Priming and the constraints it places on theories of memory and retrieval. Psychological Bulletin, 99, 650-662.

McNamara, T. (1992b). Theories of priming: I. Associative distance and lag. Journal of Experimental Psychology: Learning, Memory, \& Cognition, 18, 1173-1190.

NeELY, J. (1991). Semantic priming effects in visual word recognition: A selective review of current findings and theories. In D. Besner \& G. Humphreys (Eds.), Basic processes in reading: Visual word recog. nition (pp. 264-336). Hillsdale, NJ: Erlbaum.

NeILl, W. T., Valdes, L. A., \& Terry, K. M. (1995). Selective attention and the inhibitory control of cognition. In F. N. Dempster \& C. J. Brainerd (Eds.), Interference and inhibition in cognition (pp. 207261). San Diego: Academic Press 
Neill, W. T., Valdes, L. A., Terry, K. M., \& Gorfein, D. S. (1992) Persistence of negative priming: II. Evidence for episodic trace re trieval. Journal of Experimental Psychology: Learning, Memory, \& Cognition, 18, 993-1000.

SCHNEIDER, W. (1988). Micro Experimental Laboratory: An integrated system for IBM PC compatibles. Behavior Research Methods, Instruments, \& Computers, 20, 206-217.

Stadler, M. A., \& Hogan, M. E. (1996). Varieties of positive and negative priming. Psychonomic Bulletin \& Review, 3, 87-90.

STRAYER, D. L., \& Grison, S. (1999). Negative identity priming is contingent upon stimulus repetition. Journal of Experimental Psychol ogy: Human Perception \& Performance, 25, 24-38.

TIPPER, S. P. (1985). The negative priming effect: Inhibitory priming by ignored objects. Quarterly Journal of Experimental Psychology, 37A, 571-590.

TrPPER, S. P., \& CRANSTON, M. (1985). Selective attention and priming Inhibitory and facilitatory effects of ignored primes. Quarterly Journal of Experimental Psychology, 37A, 591-611.
Tipper, S. P., Weaver, B., \& Houghton, G. (1994). Behavioural goals determine mechanisms of selective attention. Quarterly Journal of Experimental Psychology, 47A, 809-840.

\section{NOTES}

1. The proportion of correct responses in each condition of both experiments was .98 or greater when repeated words were targets, and .94 or greater when novel words were targets. There was no indication of any form of speed-accuracy tradeoff.

2. We thank Amanda Easton, an undergraduate research assistant, for suggesting this possibility on the basis of her direct observation of the participants.
(Manuscript received January 12, 1998; revision accepted for publication March 15, 1999.) 